\title{
Remblais sur sol compressible et inclusions rigides. Amélioration de l'approche du dimensionnement
}

\section{O. COMBARIEU}

Ingénieur ESTP

HDR Université de Caen

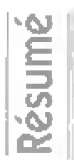

Cet article présente une étude du comportement d'un remblai frottant reposant sur un groupe d'inclusions renforçant un sol compressible. L'existence dle plans d'égal tassement vertical dans le remblai conduit à introduire, au-dessus des têtes d'inclusions. la notion de hauteur active oủ se concentrent les efforts qui leur sont transmis. Il est proposé une modification de la méthode de dimensionnement publiée en 1988. ce qui permet un calcul pius réaliste de la contrainte résiduelle en surface du sol compressible et de l'effort en tête. Les répercussions en sont examinées, quant au comportement complet du réseau et particulièrement sur l'intensité cles tassements obtenus qui constituent l'objectif recherché par le renforcement. Est étudiěe l'incidence, sur l'efficacité du qroupe, de la qualitê du matériau de remblai par le biais de son module et de son éventuelle cohésion. Le dimensionnement de la pointe des inclusions dans le sol porteur est abordê.

Mots-clés ; remblai, sol compresstble, inclusions rigides, contrainte résiduelle, effort en tête, effort global, frottement négatif, hauteur active, tassement, module, cohésion.

\section{Fill on soft soil and rigid inclusions. New design approach}

This paper presents a study of the behaviour of granular fill on compressible soil improved by a group of rigid inclusions. "The existence, in the Fill, of equal sattlement planes, leads to introduce, above inclusions heads, the notion of active height where transmitted strengths are concentrated. A modification of 1988 elesign method is proposed, which leatls to more realist residual stresses on soft soll. Incidences are examined on the whole group behaviour and particulalry on values of settlement which are the final researched objective in improvement. The quality of fill, depending of moduius and cohesion, and the inclusions tip design in bearing soil are studied.

Key words : fill, soft soil rigid thclusions, residual stress, head strength, total strength, negative skin friction, active height. settlement, modulus, cohesion. 


\section{Introduction}

Le dimensionnement d'un groupe d'inclusions rigides destiné à éliminer les tassements excessifs d'un sol compressible chargé nécessite le calcul des divers efforts, moteurs ou résistants, qui se développent tout le long de l'inclusion, depuis sa tête jusqu'à sa pointe.

La figure 1 schematise les utilisations les plus courantes d'un tel systeme de renforcement d'un sol.

L’inclusion, si elle présente une analogie évidente avec un pieu traditionnel, s'en distingue néanmoins par la nature de l'effort gui est appliqué en tểte. Pour le pieu, l'effort est transmis directement par l'intemédiaire d'une liaison rigide : c'est une donnée du projet qui conduit au dimensionnement de la fondation ; Iongueur, diamètre. Pour l'inclusion, l'effort est une inconnue, à déterminer, transmis via un mécanisme complexe, par un sol reconstitué et souple. La nature de l'effort recherché s'apparente donc à celle des efforts transmis aux pieux lors des déplacements horizontaux ou verticaux du sol autour de ceux-ci. Ce dernier cas de figure, entraînant le phénomène de frottement négatif. est à la base des méthodes de calcul des inclusions que nous avons développées (Combarieu, 1988; Combarieu, 1990).

L'intensité des efforts à calculer est três dépendante des caractéristiques du sol, de son comportement et de l'amplitude des déformations relatives. Les mécanismes d'interaction sont complexes et si les lots rhéologiques de comportement du sol peuvent aider à mieux les appréhender en utilisant le calcul aux éléments finis, la détermination de l'intensité des efforts se heurte à la difficulté d'obtention des paramètres cui caractérisent le(s) sol(s) et qu'il y a lieu d"introduire dans les modéles. Aussi des méthodes plus simples restent et resteront-elles indispensables, surtout, si confrontées à des résultats expérimentaux, elles s'avèrent pertinentes et donc suffisantes.

On se propose d'examiner dans ce qui suit le comportement du matériau d'appont (remblai, matelas) au contact de la tête d'une inclusion au sein d'un groupe. L'analyse faite conduit à proposer une amélioration de la méthode de calcul que nous avons publiée il y a vingt ans (Combarieu, 1988).

\section{Rappel du calcul de l'effort en tête et de la contrainte au sol (méthode 1988)}

On considère un groupe d'inclusions rigides verticales, à maillage carré, traversant un sol compressible et s'arrêtant sur un horizon résistant. L'ensemble est uniformément surchargé par un remblai infiniment étendu d'épaisseur constante. La notation des différentes données utilisées en 1988 est lappelée 5 ur la fịgure 2. Le côté de la maille vaut $2 \mathrm{~d}$; pour les besoins du calcul, elle est remplacée par une maille circulaire équivalente $2 b$, tel que $b / R=d / R \sqrt{\frac{4}{\pi}}$ aù $R$ est le rayon de la section draite de linclusion circulaine. Le remblai

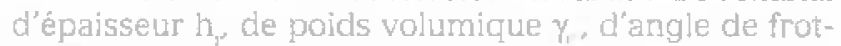
tement $\varphi_{\text {, }}$ amene avant mise en place des inclusions la contrainte $\gamma_{T} h_{r}$ en surface du sol compressible.

Ce dernier est d'épaisseur $\mathrm{H}_{\mathrm{g}}$ de poids volumique déjaugé $\gamma^{\prime}$ et est supposé saturé ('nappe en surface). Les paramètres nécessaires au calcul des efforts moteurs (frottement négatif) sont Ktan 0 et $\lambda_{r}=0$ (coefficient d"accrochage) pour le remblai et Ktan $\delta^{r}$ et $\lambda_{\text {. }}$ pour le sol.

L'effor transmis a la tête da l'inclusion au niveau $z^{*}=h_{r}$ (ou bien $z=0$ ) résulte du frottement négatif supposé s'exercer tout le long du füt d'une colonne fictive de remblai, prolongeant, sur la hauteur h, le fut de l’inclusion. Ĺadoption d'un tel mécanisme implique un déplacement relatif suffisant entre cette colonne et le remblai alentour qui accompagne le tassement du sol mou.

Dans ces conditions, le respect de l'équilibre des efforts dans un plan horizontal conduit à l'expression de la contrainte verticale dans le plan $z^{\prime}$, uniforme du fait de l'accrochage maximum $\left(\lambda_{1}=0\right)$ :

$$
q\left(z^{\prime}\right)=\frac{y_{r}}{m_{r}}\left(1-e^{\left.-m_{r} z^{\prime}\right)} \text { avec } m_{r}=\frac{2 R K \text { tanc }}{b^{2}-R^{2}}\right.
$$

A la surface du sol naturel ( $z=0$ ), entre les inclusions, la contrainte résiduelle est donc :

$$
q_{s}=q\left(h_{t}\right)=\frac{\gamma_{x}}{m_{t}}\left(1-e^{-m_{n}, r}\right)
$$
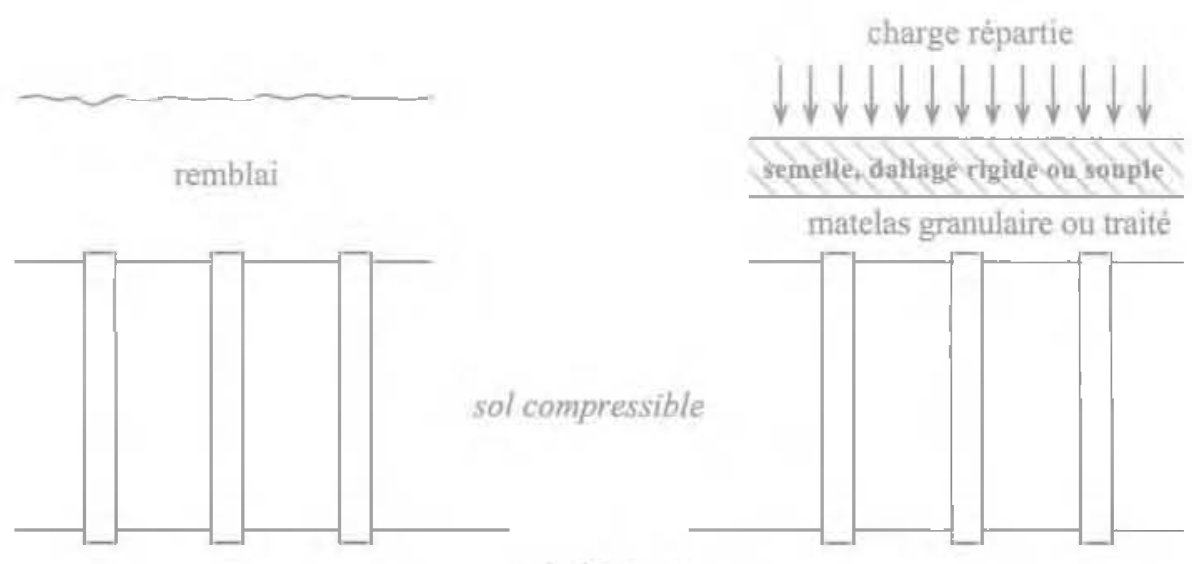

sol résistant 


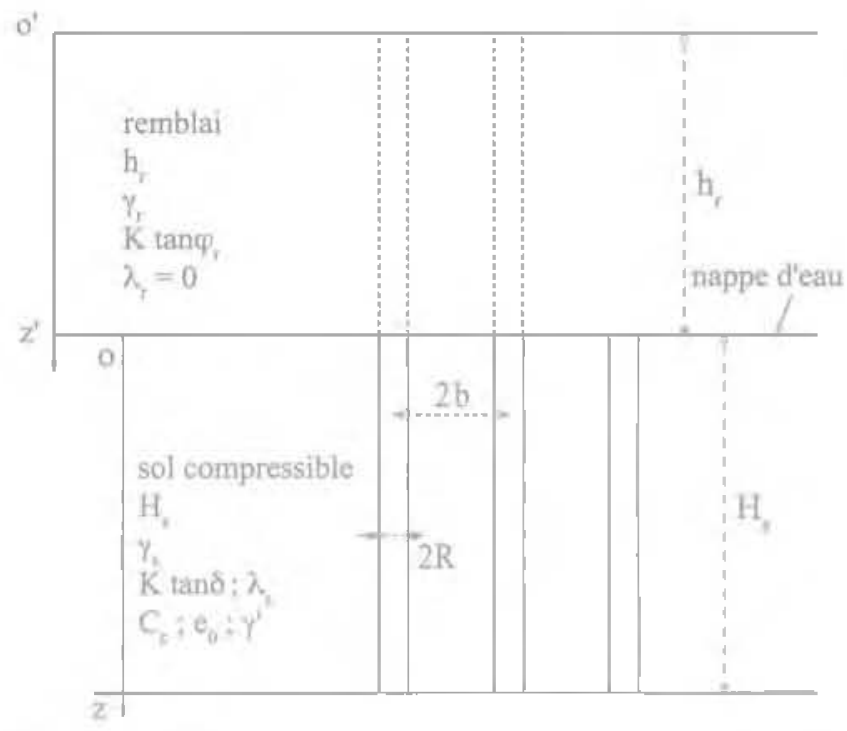

FG. Rembla sur sol renforcé par inclusions rigides (Combarieu, 1988)

Fill improvement by rigld ind usions (Combaries, 1988).

Et l'effort transmis à la tête de l'inclusion vaut:

$$
Q=\pi b^{2} y_{T} h_{T}-\pi\left(b^{2}-R^{2}\right) q\left(h_{T}\right)
$$

Pour $R$ et $K$ tan $\varphi$, fixés, l'étude des variations de $\mathrm{g}(\mathrm{h}$ ) et $\mathrm{Q}$ avec $\mathrm{b} / \mathrm{A}$ conduit aux deux courbes représentées sur la figure 3 . Pour $b=\mathrm{R}$, valeur pour laquelle il y a recouvrement complet du sol par les têtes, $\mathrm{g}\left(\mathrm{h}_{\mathrm{f}}\right)=0$ et $\mathrm{Q}=\pi \mathrm{R}^{2} \boldsymbol{\gamma}_{\mathrm{f}} \mathrm{h}_{\mathrm{r}}$. Pour b augmentant indéfiniment, (1) et (2) tendent respectivement vers $\gamma \mathrm{h}$. et

$$
Q_{\text {MAX }}=2 \pi R K \operatorname{Ran} \varphi, \gamma_{r} \frac{h_{r}^{2}}{2} \quad \text { qui est la valeur classique }
$$

connue du frottement négatif. Cette valeur $Q_{\text {Max }}$ est une borne supérieure pour la charge en tête d'une inclusion. Mais la valeur $\pi b^{7} y$ th charge d'influence pour chaque inclusion, constitue à l'évidence une borme supérieure pour $Q$ meilleure que la précédente tant que $\pi \mathrm{b}^{2} y_{\mathrm{r}} \mathrm{h}$. est inférieur' à $Q_{M A X^{\prime}} c^{\prime}$ est-à-dire tant que $\frac{b^{2}}{R^{2}}<\frac{h_{2}}{R} K \tan \phi_{r}$.

Dans la pratique des projets de rembiais de hauteur's les plus fréquentes (5 à $10 \mathrm{~m}$ ). les valeurs courantes de b/R sont faibles et la valeur de o s'avère assez proche de $\pi b^{2} \gamma h$, qui peut dans ces conditions être éventuellement choisie pour le dimensionnemert de l’inclusion.

\section{3}

\section{Proposition d'une méthode modifiée 2007}

$$
\text { L'expression (1) } q\left(h_{r}\right)=\frac{\gamma_{f}}{m_{r}}\left(1-e^{-m h}\right) \text { donne rapi- }
$$

dement une contrainte résiduelle constante $\frac{\gamma_{r}}{m_{r}}$ dès que h. est suffisamment grand, et en l'occurrence dès que,

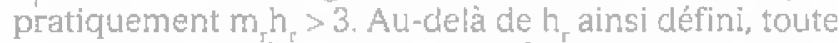
la charge supplémentaire est théoriquement reprise

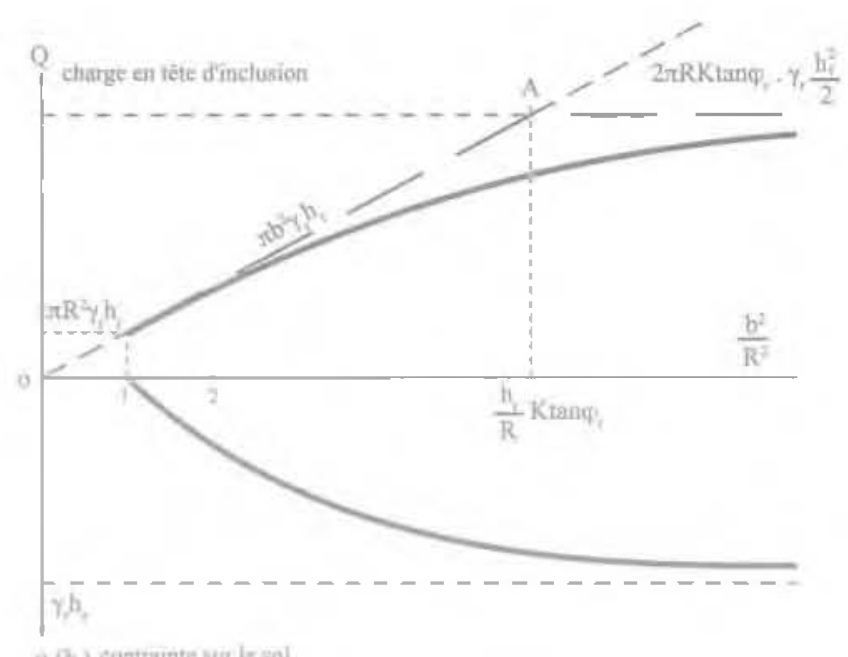

Fic. 3 Méthode 1988. Évolution, avec le maillage, de la charge en tête et de la contrainte résiduelle sur le sol

Evolution of headstrength and residual stress with inclusion spacing.

par les inclusions (dans la mesure où celles-ci sont assez résistantes). On est amene a s'interroger sur ce résultat découlant directement des hypothèses de fonctionnement adoptées et impliquant ur tassement différentiel suffisant le long de la hauteur h, des colonnes fictives. Or deux ẻléments contrarient ce principe. If n'a jamais été constaté sur chantiers réels, pour des épaisseurs h importantes, d'a empreinte $n$ des inclusions en surface du remblai terminé. En revanche, une trés faible épaisseur peut conduire à une plate-forme finale, préoccupante, en a boite à beuf's $m$. Par ailleurs la modélisation par éléments finis montre qu'à une certaine distance au-dessus des têtes, apparaît un plan d’égal tassement au-dessus duquel toute la masse de remblai descend unifornément.

Il est donc évident qu'une justification de l'effort par le développement du frottement négatif, nécessite pour les valeurs élevées de h, de limiter la hauteur le long de laquelle on convient d'un déplacement relałif suffisant. On peut ainsi introdutre une épaisseur a active $\mathrm{n} h \mathrm{~h}$. $\mathrm{h}$. sachant que pour des hauteurs de remblai reel de $7 \mathrm{~m}$. les efforts mesurés dans des inclusions assez serrés sont conformes à l'expression (2) avec 80 voire $90 \% \mathrm{de}$ la charge totale apportée. La difficulté réside dans la fixation de $h_{a}$, sachant que tant les calculs que les expérinentations laissent de côté certains aspects, non chiffrables. Ansi la mise en ceuvre des premières couches de remblai conduit, cu fait d"un compactage difficile sur du sol compressible (effet d'enclume) à un module de déformation relativement modeste ; les couches suivantes possèdent un module meilleur. La présence des têtes d'inclusions favorise pour ces premières couches la formation d'une surface en boite à oufs, déjà évoquée. Ces déformations sont compensées par la mise en place des couches suivantes qui viennent estomper les tassements différentiels. Certe compensation est bien connue dans le cas de la montée, sans renforcement, de remblai routier dont l'épaisseur centrale en fin de construction est plus forte qu'en bordure, avec une plate-forme livrée plane; on sait également que la base centrale du remblai peut être l'objet de décompression, voire de fissuration lorsquili présente de la coliésion. 
Il s'agit donc de choisir $\mathrm{h}_{\mathrm{a}^{r}}$ distance entre la tête d'inclusion et le premier plan d'égal tassement dans le corps du remblaí. L"expression retenue résulte d"abord d'une analogie avec la déformée verticale des plans horizontaux d'un massif de sol homogène sous une fondation superficielle chargée. Pour une fondation circulaire isolée de rayon $\mathrm{R}$, on peut considérer dans l'axe de celle-ci, qu'à une distance de $10 \mathrm{R}$, i] n'y a plus de déformation même avec la plastification importante sous la semelle. L'exumen détaillé de l'influence réciproque, tant en contraintes qu'en déformations. conduit par ailleurs à acmettre qu'un espacement supérieur à $6 \mathrm{R}$ élimine toute interaction. Si les tétes se rapprochent jusqu'à devenir jointives $(b / R=1)$, cas limte, la hauteur active h est évicemment rulle. On propose donc les deux expressions suivantes résultant de considérations analytiques:

$$
\frac{h_{n}}{R}=10-0,4\left(6-\frac{b}{R}\right)^{2} \text { si } \frac{b}{R} \leq 6 \text { et } \frac{h_{n}}{R}=10 \text { si } \frac{b}{R} \geq 6
$$

Cette proposition concene un matériau homogène sur toute son épaisseur et. ce, imméciatement au-dessus des têtes, reletivement ciéformable, tel celui utilisé en remblai traditionnel (avec des modules E de 50 à $150 \mathrm{MPa}$ au plus). L'interposition d'un rnatelas très raide, traité par exemple, modifie fortement les conditions d"apparition d'un plan d'egal tassement, dont la distance se trouve réduite. En effet, la grande rigidité atténue les contraintes en profondeur en les diffusant fortement: c'est le principe de la conception des chaussếes. A l'extrême, un remblai complètement traité se comporterait comme un bloc rigide très peu déformable, et avec des risques de fissuration minimes du fait de tassements faibles résultant de la présence des inclusions.

Cette expression ne contredit pas les constats expérimentaux ou obtenus par" le calcul, tels, ceux relatés dans le mẻmoire de thèse présenté récemment à l'INSA de Lyon (Jenck, 2005)

Une approche de type presslométrique peut guider également dans ja justification du choix de $h_{\text {. }}$. La valeur $Q_{\text {max }}$ obtenue pour une inclusion rigide isolée $(\mathrm{b} / \mathrm{R}=\infty)$, peut être confrontée an une estimation atutre. en considérant la tête de l'inclusion comme une plaque d'ancrage enterrée soumise à un effort d"arrachement. Cet effort vaut $k p$, où p est la pression limite dans je remblai au niveaz de la tête (Ménard, 1963; Ménard. 1969). Or on sait calculer cette pression limite si l'on connaît les caractéristiques du remblai $\left[\varphi, C_{3}, E, y_{r}\right]$. Sans entrer dans le détail de ces calculs de simulation, la comparaison montre, malgré les grandes incertitudes sur le coefficient $k$. gue l'effort d'arrachement ainsi trouvé est largement supéreur à $\mathrm{Q}_{\mathrm{MAx}}$. Cecł signifie concrètement que le choix proposé pour h $h_{a^{\prime}}$ pour linclusion isolée, est plutôt sécuritaire et qu'il en découle une efficacité du groupe sans doute un peu meilleure que celle qui résulte de la méthode proposée.

Avec l'adoption d'une hauteur active $h_{g^{\prime}}$ l'expression obtentue pour la contrainte résiduelle sur le sol compressible est dans ces conditions

$$
q_{s}=q\left(h_{s}\right)=\frac{\gamma_{r}}{m_{r}}+e^{-m r_{s}}\left[\left(h_{r}-h_{s}\right) \gamma_{r}-\frac{\gamma_{r}}{m_{r}}\right]
$$

Sur l'épaisseur h $-h_{\pi^{\prime}}$ tous les déplacements verticaux sont uniformes et Egaux et aucun frottement ne se développe au contact de la colonne fictive et du remblaj. Le frottement négatif généré par la surcharge (h- $-h_{\text {J }}$ r est reporté avec une forte intensité unitaje sur Ja hauteur active h. Pour le calcul, on compare donc h détermine par la relation (3) à la hauteur h. Si $h>h_{a}$ on applique l"expression (4), et si $h_{r}<h_{a}$ or applique (1) ou, ce quil revient an même (4) en corve nant de poser $\mathrm{h}_{\mathrm{g}}=\mathrm{h}_{\mathrm{r}} ; \mathrm{q}(\mathrm{h})$ est toujours supérieur à q ( $\mathrm{h}_{\mathrm{r}}$ ) doñé en (1).

Pour q (h. J, la charge en tête de l'inclusion vaut

$$
\mathrm{Q}=\pi \mathrm{b}^{2} \psi_{r} h_{r}-\pi\left(\mathrm{b}^{2}-\mathrm{R}^{2}\right) \mathrm{q}\left(\mathrm{h}_{\mathrm{r}}\right)
$$

Cetfe charge est toujours bornée supérieurement par la charge d"influence $\pi b^{2} y h$, lorsque $b / R$ est fai-

bile : mais la valeur $Q_{\text {max }}=2 \pi R K \tan \varphi_{r}, h_{2}\left(h_{r}-\frac{h_{a}}{2}\right)$

obtenue lorsque b/R tend vers l'infini, est la valeur naximale, inféretire à $\mathrm{Q}_{\mathrm{MAx}}$ définie précédemment. qui peut s'appliquer à la tête de l'inclusion. Cette valeur constitue la nouvelle borne qui se substitue à la charge dirnfluence dès que $\frac{b^{2}}{R^{2}}>\frac{h_{2}}{R}\left(2-\frac{h_{4}}{h_{j}}\right) K \tan \varphi_{i}$ La figure 4 illustre ce changement introduit par la méthode modifice.

\section{4}

\section{Effort en tête et contrainte résiduelle - modifications apportées par la méthode modifiée}

Lintroduction de la hauteur active diminue la charge en tête et augmente la contrainte résiduelle en surface du sol. Ces changements sont d'autant plus sensibles que l'espacement entre inclusions et que l'épaisseur h. croissent, alors qu'ils restent modérés pour les maillages serrés et des épaisseurs modérées. Le rapport SRR de la contrainte résiduelle q à $\gamma$ h, due au remblai augmente donc. Avec la méthode 1988, le rappork SRR tend vers 0 quand h. croit indéfiniment, $q$. restart constant,

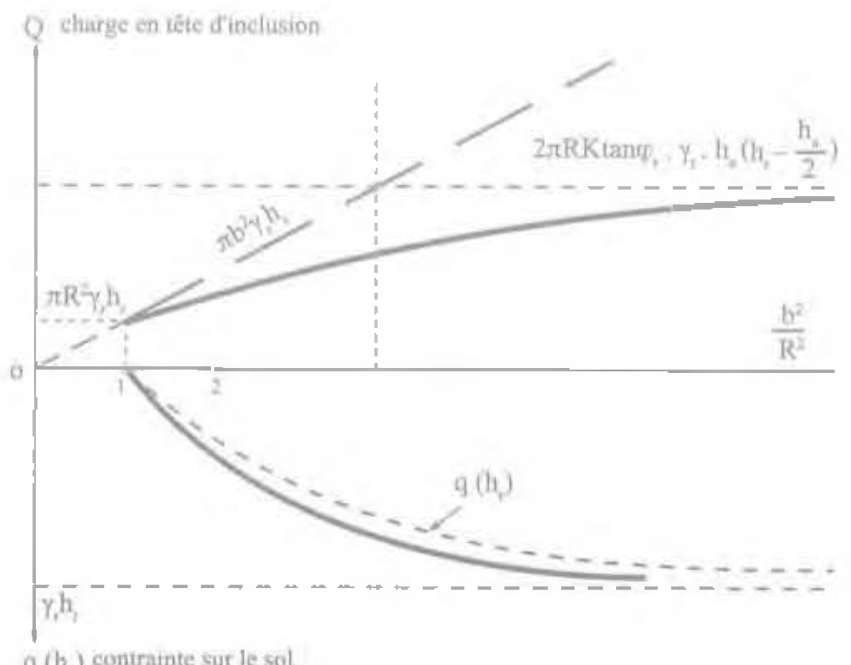
$q\left(\mathrm{~h}_{\mathrm{f}}\right)$ conirante sur le sol

FIG. Méthode 2007. Évolution, avec le maillage, de la charge en tête et de la contratinte résiduelle sur le so

Evolution of headstrength and residual stress with inclusion spacing. 
et ceci quel que soit Je maillage. La méthode 2007 donne un rapport SRR qui tend, pour b/R donné, vers $e^{-m_{s} h_{a}}$ ( $m$ dépend de $b / R$ ). limite de $\mathrm{q}_{\mathrm{s}} /\left(\gamma_{\mathrm{r}} \mathrm{h}_{\mathrm{j}}\right.$ quand $\mathrm{h}_{\mathrm{r}}$ croit.

Lefficacité, rapport de la charge en tête à la charge d'influence, varie toujours de 1 pour $b / R=1$, à 0 pour b/R infini, maís sa valeur est plus faible avec la méthode modifiée.

Le rapport de concentration n est le rapport entre contrainte en tête et contrainte résiduelle, qui varient en sens inverse ; il ne présente, de ce fait, qu'un intêrët restreint. Il est intini pour b/A $=1$, ce qui colrespond à une efficacitế maximale, il décroit très rapidement et tend pour h. infini vers la valeur limite finie

$n=1+\frac{b^{2}}{R^{2}}\left(e^{m / 2}-1\right)$; auparavant, cette linite était infinie et ce quel que soit le maillage.

\section{5}

\section{Charge totale dans l'inclusion}

Les répercussions d"une baisse de la contrahte résiduelle $q_{\text {s }}$ sonl simples. Laction bénéfìque de la présence des inclusions se poursuit en effet au sein du sol mou par l'apparition du frottement négatif $F_{\text {n }}$ le long de celles-ci, généré par l'action de cette contrainte résiduelle, augmentant de $\mathrm{q}\left(\mathrm{h}_{\mathrm{r}}\right) \mathrm{à} \mathrm{q}\left(\mathrm{h}_{\mathrm{n}}\right)$. Ce frottement F augmente donc lui aussi et compense, partiellement, la baisse concomitante de la charge en tête. En définitive, la charge totale dans l'inclusion, cumulant la charge en tête et le frottement négatif, est plus faible avec la proposition de modification, et son point d'application (le point neutrel se situe un peu plus bas dans l'inclusion (dans la mesure oủ il ne se situe pas dejà au bas de la couche de sol mou).

La charge totale est toujours bornée par la charge d'influence $\pi \mathrm{b}^{2} \gamma_{\mathrm{r}} \mathrm{h}_{\mathrm{r}}$ Elle ne peut néanmoins dépasser une borne maximale, somme de la bome donnèe en (2) ou en (6) et du frottement négatif dû à $q$ (h.) ou $\mathrm{g}(\mathrm{h})$ sur une inclusion rigide isolée $(\mathrm{b} / \mathrm{R}=\infty$ ) au sein du sol mou. Une borne pour $F_{\text {mak }}$ est donnée par $2 \pi R K \tan \delta\left(\gamma_{,} h_{1} H+\gamma \frac{H^{2}}{2}\right)$, donnant une valeur un peu supërieure à la véritable borne, car, par simplification, il a été pris en compte un accrochage maximal $\left(\lambda_{5}=0\right)$.

Ces bornes n'ont qu'un intèrêt mineur, puisqu'elles correspondent d̀ des maillages très grands, sans utilité pratique pour" la conception de groupes d'inclusions.

On peut certes se contenter pour un calcul de groupe, d'un dimensionnement ameêté au niveau des têtes, et calculer le tassement de la couche compressíble sous la seule contrainte $\mathrm{g}$ (h) en négligeant l'effet bénéficue des inclusions. C'est en effet la valeur du tassement absolu ou différentiel, souvent les deux, qui est imposée comme objectif à atteindre : cette valeur constitue donc l'arbitre final pour juger de la pertinence de la géométré du groupe à laquelle on est parvenu. Aussi, mème si le frottement négatif $F_{m=}$ le long des inclusions reste modeste vis-à-vis de la charge en tête.

"Pour des leasons liées aux exptessions mathematinges de h, et mo"

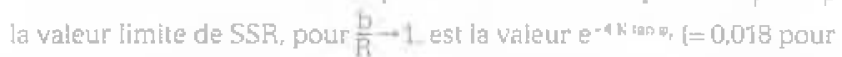

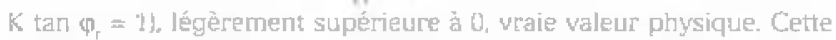

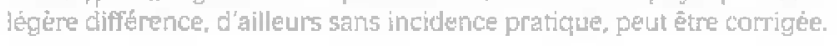

son effet de réduction de la contrainte verticale induite dans le sol compressible, contribue à une réduction supplémentaire non négligeable du tassement dont on aurait tort de se priver. L'exemple numérique suivanten. fait la démonstration. On dispose d'ailleurs de règles de calcul des efforts de frottement négatif quj bénéficient d'une sanction expérimentale solide quant aux valeurs du terme $\mathrm{K}$ tano essentiel au calcul ; les calculs de tassement qui en découlent sont donc parfaitenent fondés. Comme $q\left(h_{a}\right)>q\left(h_{r}\right)$, le tassement final s'avère un peu plus élevé. La détermination de $F_{n s}$ relève toujours de le méthode 1988 qui n'est l'objet d'aucun changement en ce cuil concerre le sol compressible

\section{6}

\section{Exemple numérique}

Afin d'illustrer les developpements qui viennent d'être exposés, on a choisi le cas d'application d'un remblai, routier ou ferroviaire, pouvant atteindre des hauteurs importantes et reposant sur du sol compressible.

Pour le remblai, les données sont les suivantes : $h_{r}=5,10$ et $15 \mathrm{~m} ; \gamma_{\mathrm{r}}=20 \mathrm{kN} / \mathrm{m}^{3} ; \varphi_{\mathrm{r}}=40$ degrés; $K \tan \varphi_{r}=0,9$

Pour le sol compressible, il s'agit d'une argile dont les caractéristiques sont les suivantes:

$\mathrm{H}_{\mathrm{t}}=5 \mathrm{~m} ; \gamma=8 \mathrm{kN} / \mathrm{m}^{3} ; \mathrm{e}_{0}=1,15 ; \mathrm{C}=0,35 ; \mathrm{C}_{\mathrm{5}}=0,05$. La nappe varie régulièrement entre la surface du sol (niveau 0) et $-1 \mathrm{~m}$.

Les inclusions ont $0,40 \mathrm{~m}$ de dlametre et trois maillages sont étudiés pour le groupe avec b/R valant respectivement 2,5 : 4 et 6 . Elles sont de type foré avec, en conséquence, les paramètres de contact sol-inclusion : $\mathrm{K} \tan \delta=0,15$ et $\lambda$, coefficient d'accrochage égal 2. 0,235 (les relations liant $K$ tan $\delta$ et $\lambda$ sont données dans la méthode 1988, citée en références).

On considère clans cet exemple que la résistance intrinsèque des inciusions est toujours suffisante.

Dans les tableaux I à III sont donnés les résuiltats détaillés pour les deux modes de calcul : méthode initiale de 1988 et méthode modiflée de 2007 . Ces résultats permettent toutes les comparaisons souhaitées entre les deux approches. La figure 5 donne les courbes d'évolution du rapport SRR avec l'épaisseur h pour les trois maillages étudiês. Eilles illustrent les différences introduites par les conditions nouvelles lon y a reporté les valeurs calculées pour une hauteur de remblai de 2 mi

La figure 6 donne, pour la méthode 2007 , les valeurs absolues des tassements moyens en surface du sol mou, tenant compte ou non de l'effet du frottement négatif le long du fût des inclusions, ainsi que celles obterues sans inclusions $(b / R=\infty)$. Cet effet ne peut être négligé, son influence sul" la réduction des tassements saverant importante.

\section{7}

\section{Le tassement du sol et des inclusions}

Les tassements excessifs qu'accuserait le massif compressible sous le poids d'un remblaj constituent une des raisons, essentielle, qui amene à renforcer ce massif par 


\begin{tabular}{|c|c|c|c|c|c|c|}
\hline Maillage b/R & 1 & 2.5 & 4 & 6 & $\infty$ & \\
\hline 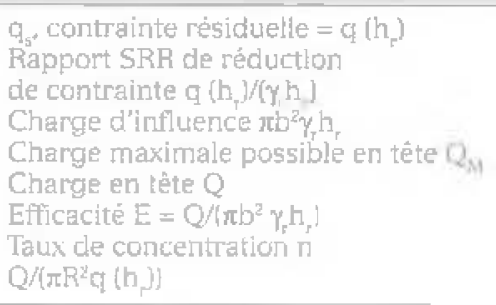 & $\begin{array}{l}0 \\
0 \\
13 \\
13 \\
1 \\
\infty\end{array}$ & $\begin{array}{c}12 \\
0.12 \\
79 \\
71 \\
0.90 \\
48\end{array}$ & $\begin{array}{l}32 \\
0.32 \\
201 \\
141 \\
0.70 \\
35\end{array}$ & $\begin{array}{c}56 \\
0,56 \\
453 \\
204 \\
0,45 \\
29\end{array}$ & $\begin{array}{c}100 \\
1 \\
282 \\
282 \\
0 \\
22\end{array}$ & $\begin{array}{l}\text { méthode } \\
198 B\end{array}$ \\
\hline $\begin{array}{l}\text { Frottement négatif F } \\
\text { Point methe } h_{L}(\mathrm{~m})\end{array}$ & $\begin{array}{l}0 \\
0\end{array}$ & $\begin{array}{c}6 \\
2,20\end{array}$ & $\begin{array}{l}36 \\
5,0\end{array}$ & 58 & & $\begin{array}{l}\text { mẻthode } \\
1988\end{array}$ \\
\hline $\begin{array}{l}\text { Charge totale } Q_{9} \\
\text { Efficacité totale } \mathrm{E}=\mathrm{Q} /\left(\pi \mathrm{b}^{2} \gamma_{\mathrm{g}} \mathrm{h}_{\mathrm{r}}\right)\end{array}$ & $\begin{array}{l}13 \\
1\end{array}$ & $\begin{array}{l}77 \\
0,97\end{array}$ & $\begin{array}{l}177 \\
0,88\end{array}$ & $\begin{array}{c}262 \\
0,58\end{array}$ & 0 & \\
\hline 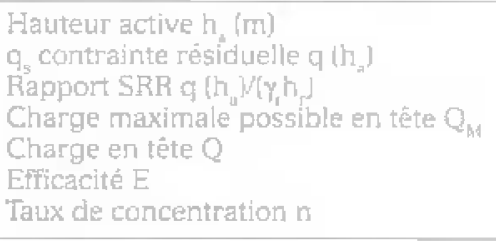 & $\begin{array}{c}0 \\
0 \\
13 \\
13 \\
1 \\
0\end{array}$ & $\begin{array}{l}1.02 \\
24 \\
0.24 \\
102 \\
63 \\
0.79 \\
21\end{array}$ & $\begin{array}{c}1,68 \\
45 \\
0,45 \\
158 \\
115 \\
0,57 \\
20\end{array}$ & $\begin{array}{c}2,0 \\
67 \\
0,67 \\
181 \\
157 \\
0,35 \\
19\end{array}$ & $\begin{array}{c}100 \\
1 \\
181 \\
0 \\
14\end{array}$ & $\begin{array}{l}\text { méthode } \\
\text { 2007 }\end{array}$ \\
\hline $\begin{array}{l}\text { Frottement régatif } F_{\text {na }} \\
\text { Point neutre } \mathrm{h}_{\mathrm{E}} \text { (mT) }\end{array}$ & 0 & $\begin{array}{l}13 \\
3,0\end{array}$ & $\begin{array}{r}45 \\
5,0\end{array}$ & $\begin{array}{l}67 \\
5,0\end{array}$ & & $\begin{array}{c}\text { méthode } \\
1988 \\
\text { [sans } \\
\text { changement) }\end{array}$ \\
\hline $\begin{array}{l}\text { Chauge totale } \mathrm{Q}_{1} \\
\text { Efficacite totale } \mathrm{E}=\mathrm{Q} /\left(\pi \mathrm{b}^{2} \psi_{1} \mathrm{~h}\right)\end{array}$ & $\begin{array}{c}13 \\
1\end{array}$ & $\begin{array}{c}76 \\
0,96\end{array}$ & $\begin{array}{l}160 \\
0,80\end{array}$ & $\begin{array}{c}224 \\
0,49\end{array}$ & 0 & \\
\hline
\end{tabular}

Les contraintes sont exprimëes en kPa êt tes charges ou efforts en $\mathrm{kN}$.

TA:LEAU II $h_{-}=10 \mathrm{~m} ; \gamma_{r} h_{r}=200 \mathrm{kPa}$.

\begin{tabular}{|c|c|c|c|c|c|c|}
\hline Maillagu b/R & 1 & 2,5 & 4 & $b$ & $\infty$ & \\
\hline 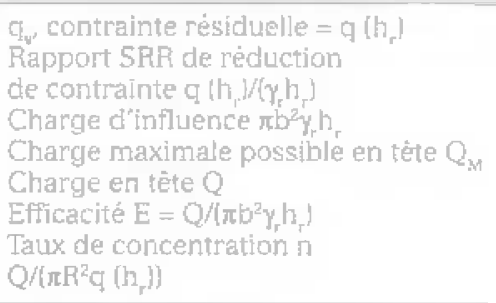 & $\begin{array}{l}0 \\
0 \\
25 \\
25 \\
1 \\
00\end{array}$ & $\begin{array}{c}12 \\
0,06 \\
157 \\
149 \\
0,95 \\
98\end{array}$ & $\begin{array}{l}33 \\
0.17 \\
402 \\
339 \\
0.84 \\
82\end{array}$ & $\begin{array}{l}72 \\
0.36 \\
905 \\
588 \\
0,65 \\
65\end{array}$ & $\begin{array}{l}200 \\
1 \\
\infty \\
1130 \\
1130 \\
0 \\
45\end{array}$ & $\begin{array}{l}\text { méthode } \\
\text { Tage }\end{array}$ \\
\hline 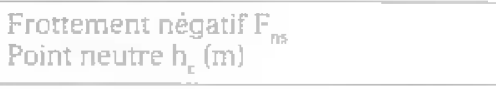 & 0 & $\begin{array}{c}6 \\
2,2\end{array}$ & 37 & $\begin{array}{l}71 \\
5\end{array}$ & & $\begin{array}{l}\text { methode } \\
1988\end{array}$ \\
\hline $\begin{array}{l}\text { Charge totale } \mathrm{Q} \\
\text { Efficacité totale } \mathrm{E}=\mathrm{Q} /\left(\pi b^{2} \gamma_{r} \mathrm{~h}_{\mathrm{r}}\right)\end{array}$ & $\begin{array}{c}13 \\
1\end{array}$ & $\begin{array}{r}155 \\
0,99\end{array}$ & $\begin{array}{c}376 \\
0.94\end{array}$ & $\begin{array}{l}659 \\
0.73\end{array}$ & 0 & \\
\hline 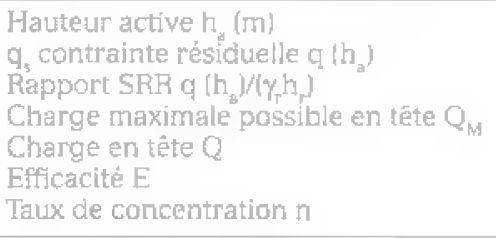 & $\begin{array}{c}0 \\
0 \\
25 \\
25 \\
1 \\
5\end{array}$ & $\begin{array}{l}1,02 \\
42 \\
0,21 \\
215 \\
129 \\
0,82 \\
24\end{array}$ & $\begin{array}{c}1,68 \\
82 \\
0,41 \\
348 \\
248 \\
0,62 \\
24\end{array}$ & $\begin{array}{c}2,0 \\
127 \\
0,63 \\
407 \\
346 \\
0,38 \\
22\end{array}$ & $\begin{array}{c}200 \\
1 \\
407 \\
0 \\
16\end{array}$ & $\begin{array}{l}\text { méthode } \\
2007\end{array}$ \\
\hline $\begin{array}{l}\text { Frottement négatlf } F_{m s} \\
\text { Point neutre } h_{c}(\mathrm{~m})\end{array}$ & 0 & $\begin{array}{l}21 \\
3,5\end{array}$ & $\begin{array}{c}69 \\
5\end{array}$ & $\begin{array}{c}112 \\
5\end{array}$ & & $\begin{array}{c}\text { rnéthode } \\
1988 \\
\text { (sans } \\
\text { changennent) }\end{array}$ \\
\hline $\begin{array}{l}\text { Charge totale Q } \\
\text { Efficacite totale } E=Q_{1} /\left(\pi b^{2} \gamma_{\mu} h_{n}\right)\end{array}$ & $\begin{array}{c}25 \\
1\end{array}$ & $\begin{array}{r}1,50 \\
0,96\end{array}$ & $\begin{array}{c}317 \\
0,79\end{array}$ & $\begin{array}{c}458 \\
0.51\end{array}$ & 0 & \\
\hline
\end{tabular}

Les contraintes sont exprimées en $\mathrm{kPa}$ et les charges ou efforts en $\mathrm{kN}$. 


\begin{tabular}{|c|c|c|c|c|c|c|}
\hline Maillage b/R & t & 2,5 & 4 & B & $\mathrm{sat}$ & \\
\hline 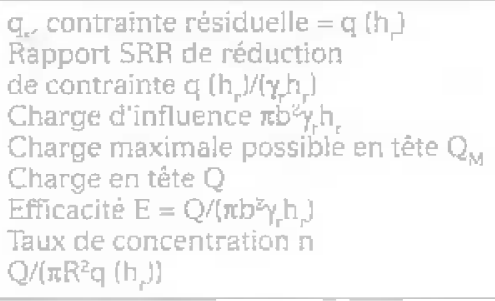 & $\begin{array}{c}0 \\
0 \\
50 \\
50 \\
1 \\
0\end{array}$ & $\begin{array}{l}12 \\
0,03 \\
314 \\
306 \\
0,97 \\
203\end{array}$ & $\begin{array}{c}33 \\
0,08 \\
805 \\
740 \\
0,96 \\
178\end{array}$ & $\begin{array}{l}77 \\
0.19 \\
1810 \\
1457 \\
0,85 \\
152\end{array}$ & $\begin{array}{c}400 \\
1 \\
4522 \\
0 \\
90\end{array}$ & $\begin{array}{l}\text { méthode } \\
1988\end{array}$ \\
\hline $\begin{array}{l}\text { Frottement thégatif } F_{m} \\
\text { Point neutre } h_{s}[m]\end{array}$ & 0 & $\begin{array}{l}6 \\
2,2\end{array}$ & $\begin{array}{l}37 \\
5\end{array}$ & $\begin{array}{c}74 \\
5\end{array}$ & & $\begin{array}{l}\text { méthode } \\
1988\end{array}$ \\
\hline $\begin{array}{l}\text { Charge totale } Q_{1} \\
\text { Efficacite totale } E=Q_{1} /\left(\pi b^{2} \gamma_{p} h_{\uparrow}\right)\end{array}$ & $\begin{array}{c}50 \\
1\end{array}$ & $\begin{array}{r}312 \\
0.99\end{array}$ & $\begin{array}{l}7 \overline{7} \\
0,96\end{array}$ & $\begin{array}{l}1541 \\
0,85\end{array}$ & 0 & \\
\hline 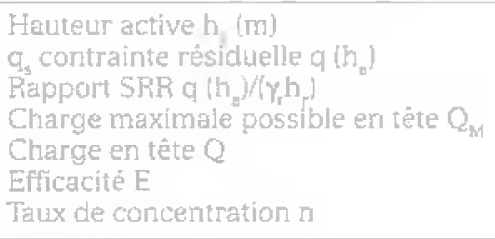 & $\begin{array}{l}0 \\
0 \\
50 \\
1 \\
0\end{array}$ & $\begin{array}{l}1,02 \\
78 \\
0,19 \\
441 \\
283 \\
0,84 \\
27\end{array}$ & $\begin{array}{c}1,68 \\
155 \\
0,39 \\
728 \\
512 \\
0,64 \\
26\end{array}$ & $\begin{array}{c}2,0 \\
247 \\
0,62 \\
859 \\
725 \\
0,40 \\
24\end{array}$ & $\begin{array}{c}400 \\
859 \\
859 \\
0 \\
17\end{array}$ & $\begin{array}{l}\text { méthode } \\
2007\end{array}$ \\
\hline $\begin{array}{l}\text { Frottement négatif }{ }^{2} \\
\text { Point neutre } h_{\Sigma}(m)\end{array}$ & 0 & $\begin{array}{l}43 \\
4,7\end{array}$ & $\begin{array}{c}118 \\
5\end{array}$ & $\frac{202}{5}$ & & $\begin{array}{c}\text { méthode } \\
\text { 1.988 } \\
\text { (5ans } \\
\text { changement }\end{array}$ \\
\hline $\begin{array}{l}\text { Charge totale } \mathrm{Q} \\
\text { Efficacité totale } \mathrm{E}=\mathrm{Q}_{1} /\left(\pi \mathrm{b}^{2} \mathrm{\gamma}_{r} \mathrm{~h}_{r}\right)\end{array}$ & $\begin{array}{c}50 \\
1\end{array}$ & $\begin{array}{c}307 \\
0.98\end{array}$ & $\begin{array}{c}630 \\
0.78\end{array}$ & $\begin{array}{l}927 \\
0,51\end{array}$ & 0 & \\
\hline
\end{tabular}

Les contraintes sont exprimées en $\mathrm{kPa}$ et les charges ou efforts en kN.

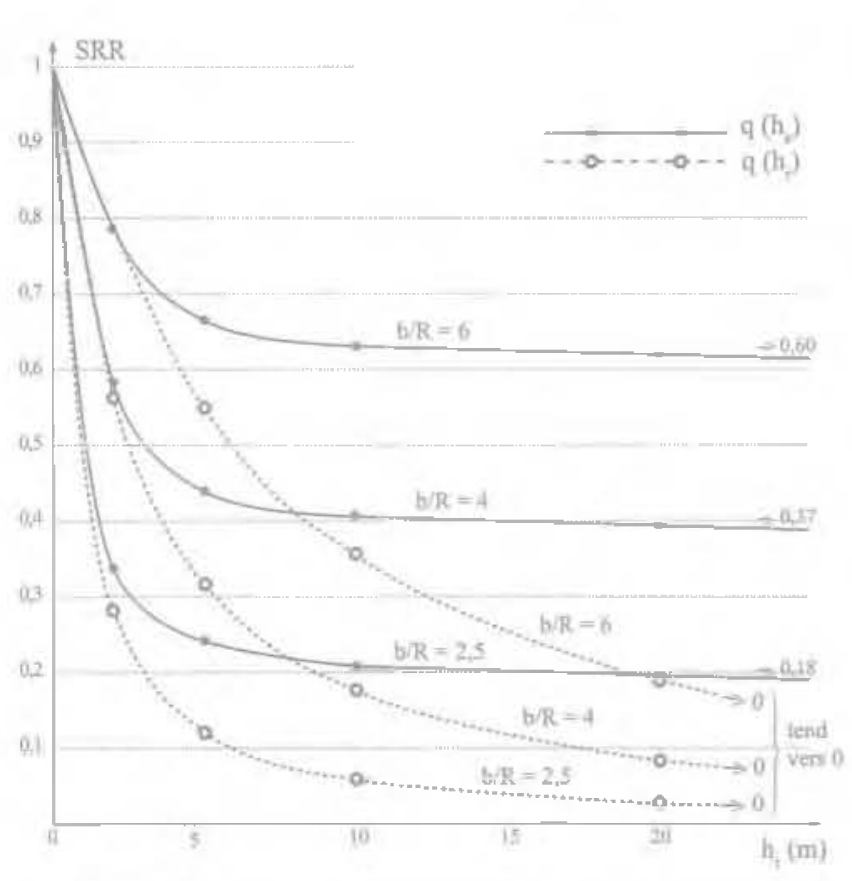

FG. 5 Evolution du rapport SSR en fonction de h. Evolution of SSP ratio with $h_{\text {. }}$ inciusions rigides. On cherche à réduire fortement les déformations en les ramenant à des valeurs imposées, de même qu'on cherche à construire rapidement en éliminant les problèmes de stabilité. Il est donc important de prévoir au mieux ces tassements, totaux, différentiels, différés et de garantir l'obtention des valeurs fixées.

Ces tassements sont le résultat d'une réduction de la surcharge de remblai par la mobilisation simultanée d'un effort en tête d'inclusion et du frottement réga-

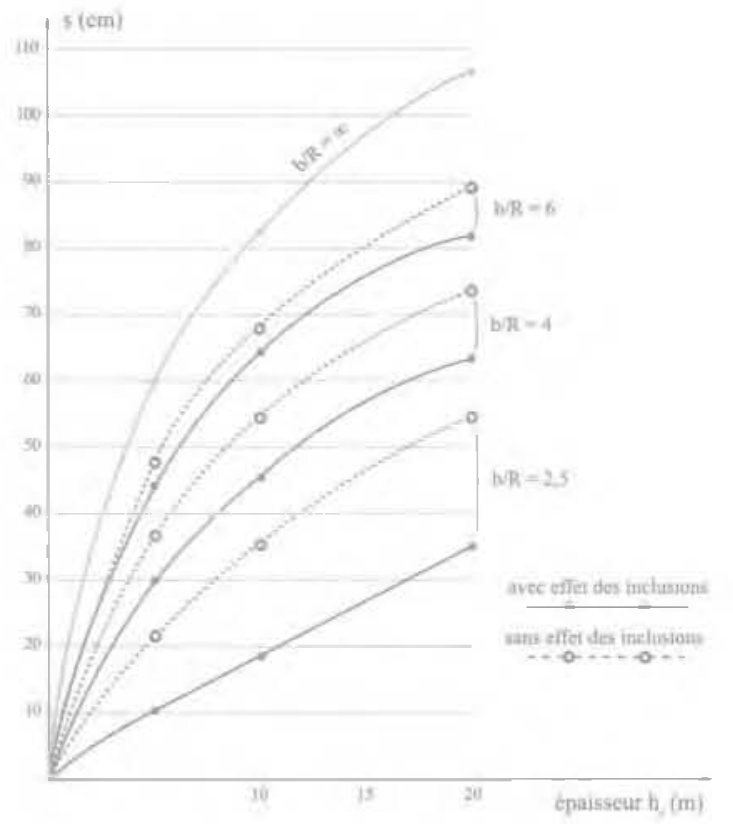

Fต. 6 Évolution du tassement sous $\mathrm{q}_{5}=\mathrm{q}\left(\mathrm{h}_{\mathrm{g}}\right)$ en fonction de $h_{\text {. }}$

Evolution of seltlement under $q_{5}=q\left[h_{n}\right]$ with $h_{r^{\prime}}$

tif sur une fraction ou la totalité du fût de celle-ci. La charge totale Q, somme de ces deux efforts, doit être équilibrée par l'effort offert par la fiche résistante dans l'horizon porteur sous-jacent, et le frottement positif mobilisé dans la partie inférieure de l'inclusion, située au-delà du point neutre. Ce frottement sera souvent unitairement faible, du fait des caractéristiques, ellesmèmes faibles, du sol mou, et de l'influence de l'effet de groupe. 
A ce stade du dimensionnement, deux choix extrế mes sont possibles. Certains concepteurs, dans l'optique d'une inclusion a fixe n en pointe, préconisent une sécurité de 2 ou 3 sur la charge limite de la fiche ; le fonctionnement est identique à celui d'une fondation profonde traditionnelle. Cela assure a priori des déformations relatives suffisantes entre sol et inclusion poun justifie" le calcul des efforts moteurs. Cette sécuritẻ parait cependant excessive et certains, dont l'auteun de cet article, recommandent de la réduire fortement. en admettant de faire travailler la partie résistante à sa charge de fluage, voire sa charge lirnite.

Dans le premier cas, avec des pointes fixes, on dispose d'une réserve d'effort, permettant, au cours de la vie de l'ouvrage, l'ajout en tête de rembiai d'une surcharge nouvelle. Cette dernière se repartit entre sol mou et inclusion, r'entrainant qu'un tassement modéré dont on s'assure qu'il peut être acceptable. La répartition étant calculable, on accède à la valeur maximale cle cette surcharge conduisant progressiveritent la fiche résistante à sa charge limite.

Dans le second cas, l'application de la même surcharge nouvelle sollicite intégralement fe sol mou sur toute son épaisseur ; l’inclusion ne peut reprendre aucur effort supplémentaire, et la surface du sol mou et la tête dinclusion accusent un même tassement, supertieur au précédent.

Cependant, lincertitude sur le calcul de la charge limite de la fiche résistante et les diffémences, quelquefois faibles auxquelles on parvient sur la longueur de cette fiche, peuvent faire que cette alternative n'a en réalité guère d'intêrêt ; c'est particulièrement vTà si la longueur totale de l'inclusion est forte. La frange supérieure de l’horizon porteur est aussi un élément a considérer, car son irrégularité en plan, son altération, son remaniement sont autant de facteurs quí vont rendre necessaire l'obtention d'une fiche minimale. A l'inverse, la présence continue d'un niveau rocheux permet d'y poser simplement les pointes.

Un autre aspect doit être évoqué, il s'agit de l'effet de groupe. On en tire profit pour justifier le dimensionnement du réseau, mais on le néclige ou l'oublie au niveau du sol porteur ou i] a d'autant plus de raison d'être pris en compte que le maillage est serre. Dans l'exemple numérique traité, le maillage de $b / \mathrm{H}=$ 2,5 conduit à une efficacité totale de plus de $95 \%$, la charge totale étant appliquée pratiquernent au niveau du sol porteur. Dans ces conditions, que la pointe des inclusions soit fixe ou mobile, le tassement du groupe dépend de la compressibilité de I'horizon porteur et diffère assez peu d'un cas à l'autre dans la mesure où l’horizon est homogene. La différence n’atteint pas celle obtenue entre le tassement d'un pieu à sa charge limite, cle l'ordre du dixième du diamètre. et celuj d'un pieu où la sêcurité est de 2 à 3. de l'ordre du centième du diamètre de celui-ci. Poup les inclusions les plus couramment réalisées. d'un diamètre de l'ordre de $40 \mathrm{~cm}$, ces valeurs restent de toute manjère faibles, avec 4 ä 5 crm pour la plus torte. C'est en ces termes que se poserait le calcul du tassement dans le cas de figure, extrême et totalement irréaliste, d'un groupe d'inclusions qui seraient toutes jointives et indépendantes, recouvrant totalement la surface du sol mou; on imaginerait alors des füts de plus faible section droite que la tête et l'ensemble serait similaire à celui d”une dalle sum pieu. Les groupes efficaces ne sont pas très éloignés de cette configuration.
Enfin. il est essentiel de rappeler que les déformations ne sont gênantes que si elles sont préjudiciables au bon fonctionnement des ouvrages en service. Par exemple, pour les remblais ferroviaines, les exigences imposées par l'exploitant ne concernent que les déformations à venir après mise en place de la voje.

Aussi, dans la mesure oủ les tassements, qui seraient obtenus sans renforcement, sont d'intensité suffisante, cet ensemble d'éléments permet d'affirmer que le problème du cholx de pointes d'inclusions a fixes a ou u mubiles a n'est te plus souvent pas important et les tassements qui se manifestent au niveau des pointes sont le plus souvent rapides et non rédhibitoires.

Afin de préciser ce que sont des tassements d"intensité suffisante, il y a lieu d'éwoguar le cas de la très faible déformabilité du massif pour lecuel il sera très pet courant, pour des remblais qui y son construits. d'utiliser des inclusions rigides. La méthode gènérale de dimensionmement exposée ne fait appèl qu'à des conditions de contraintes limites : elle admet implicitement que les deformations relatives entre le sol ef le fût de l'inclusion et entre le remblai [ou mateles) et la tête de l'inclusion sont suffisantes pour mobiliser" les contraintes. C'est le cas de très nombreuses situations. On sait que pour le füt. le déplacement relatif́ nécessaire et suffisant est de l'ordre de $1 / 100$ du diamètre 2 R du füt; pour la tête, même si l’on fait appe! au phénomène de frottement négatif pour justifier le dimensionnement, c'est en réalité le poinçonnement dans le matelas qui es: mis en cause. La capacité limite demande donc (et c'est l'analogie avec le poinçonnment d'une semelle qui permet cette hypothèsel une déformation relative de $1 / 10$ du diametre $2 \mathrm{R}^{\prime}$ de cette tête, la moitié environ de cette capacité ne nécessitant que $1 / 100$ de $2 \mathrm{R}^{\prime}$

Si le sol s'avère peu compressible et ne mène sans traitement qu'à de faibles tassements même sous forte charge, on ne peut appliquer brutalement la méthode sans risque d'un surdinensionnement du groupe d'inclusions auquel elle mène; on arriverait ainsì au cas extrême d'un sol quasiment indéformable que l'on renforcerait, alors que c’est bien sûr inutile.

Aussi, avant toute décision de renforcement et, plus généralement, d'amélioration, il est impératif de procéder à l'étude des déformations et plus précisément à l'étude du profil vertical $\mathrm{S}$ (2) des tassements sur l'êpaisseur $\mathrm{H}_{s}$ du massif concerné.

Le critère de renforcemen étant la déformée ver ticale en surface, la udensité o de renforcement est directement líe à la différence entre le tassement sans renforcement $\mathrm{S}$ et le tassement admissible S à obtenir sous charge après renforcement. La densité du groupe est nulle si $\mathrm{S}-\mathrm{S}_{\mathrm{n}}=0$.

Aussi. dans la mesure où les tassements sans renforcement sont petits, il est de loin préférable de " fixer $x$ les pointes pour favoriser le déplacement relatif requis pour mobilliser les contraintes.

On peut dailleurs constater que plus $S$ - S baisse, plus la densité des renforts baisse; si on impose la longueur des inclusions, on est amené à des diamètres de plus en plus faibles.

Une remarque simpose également quant aux tas. sements attendus. Tels que nous les avons calculés, ils sont liés à la consolidation et se manifestent théoriquement plus ou moins lentement. Or, on constate souvent que les tassements acquis, faibles, le sont rapidement. On ne peut s'empêcher de faire le raporochement avec 
un comportement élastique instantané, cénéralement négligé, car de faible amplitude. La surconsolidation peut fournir une explication à ce constat. Elle existe souvent, ne serait-ce que par la variation de la nappe d'eau superficielle, associée à une couche de surface de meilleure qualitè que l'ensemble du massif. Cotte influence est donc réelle, précisément dans le domaine des faibles contraintes résiduelles en surface, et négliger ou ignorer cette réalité physique de la surconsol: dation n'est pas sans enjeu sur le dimensionnement.

\section{8}

\section{Influence de la qualité du remblai}

La performance du renforcement est trés dépendante de la qualité du remblai, plus spécialement sur la partie inférieure de celui-ci, la hauteur active $\mathrm{h}_{\mathrm{H}}$ qui mobilise les efforts transmis a la tête. En effet, le terme $\mathrm{K}$ tan $\varphi_{\mathrm{t}}$ est directement lié à cette qualité et c'est lui qui détermine, pour $\mathrm{b} / \mathrm{R}$ donné, la contrainte résiduelle $\mathrm{q}$. et l'effort en tête. Airisi si $K \tan \varphi$, est nul, $q_{5}$ vaut $\psi_{\text {h }}$ et l'effort est nul (hormis le poids de la colonne de remblai). Par ailleurs la valeur limite $e^{-m_{1} h_{a}}$ du rapport SRR, pour $h_{F}=\infty$ comporte le terme :

$m_{h} h_{s}=\frac{10-0,4\left(6-\frac{b}{R}\right)^{2}}{\frac{b^{2}}{h^{2}}-1} K \operatorname{can} \varphi_{e}$ proportionnel a $K$ Lan $\varphi_{\mathrm{r}}$

Le choix de la valeur de $\mathrm{K} \tan \varphi_{\mathrm{T}}$ est donc essentiel et la qualité finale du remblas dont ce teme dépend doit être fixée par des spécifications à bien respecter. Les documents de Recommandations existent, auxquels on se référera (LCPC-COPREC, 1980; SETRALCPC, 1992)

Dans ce domaine, les réceptions de corps de remblai ou de plates-formes sont très souvent basées sur la mesure des modules de défomation, mesurés, soit par essai statique de plaque, soit par impact a ta dynaplaque ou au portancemètre. Ces materiels foumissent des valeurs de module corrêlées entre elles ou directement prescrites dans les spécifications pour un appareillage donné. Toutefois, l'obtention des modules demandés est également tributaire, pour les toutes premieres couches de remblai mises en cuvre, de la rigidité du sol support. Il est illusoire d"espérer bien compacter sur un sol mou, ce qui nécessite souvent de sacrifier une première épaisseur, indispensable d'ailleurs à la circulation des engins; ; i] est done reconmandé de travailler en période d'étiage en profitant tamleau N. Valeurs de $\mathrm{K}$ tan $p_{\mathrm{r}}$ en fonction du module $\mathrm{E}$.

\begin{tabular}{c|c|c|c|c|c|cc}
\hline E(MPa) & 20 & 30 & 40 & 50 & 70 & 100 & 150 \\
\hline $\mathrm{K} \tan \varphi_{\mathrm{r}}$ & 0,43 & 0,55 & 0,65 & 0,73 & 0,85 & 0,96 & 1,05 \\
\hline
\end{tabular}

de la croûte superficielle moins déformable. Enfin, le module obtenu après compactage de la couche, augmente très sensiblement après que cette couche a été recouverte par les couches suivantes; ce constat, logique, a pu être fait sur des remblais.

Pour le calcul, il s'agit, le module E étant connu, d'en déduire la valeur K tar ø à utiliser, sachant que la nature et la granulométrie du matériau sont des éléments â prendre en compte. Le mode et les paramètres de compactage ont eux-mênes une influence directe tant sur l'angle $\varphi$, que sur le coefficient $K$ tan $\varphi_{\text {. On }}$ propose la relation empirigue

$$
K \tan \varphi_{\mathrm{r}}=1.1-\mathrm{e}^{-E E_{\mathrm{s}}}
$$

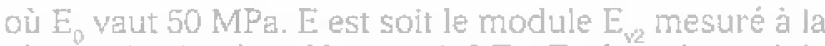
plaque de diamètre $60 \mathrm{~cm}$, soit $3 \mathrm{E}_{\mathrm{M}, \mathrm{E}_{\mathrm{M}}}$ étant le module mesuré au pressiomètre Ménard. Cette relation fournit les valeurs du tableau IV, cohérentes avec celles données pour $K \tan \varphi$, dans le fascicule 62, titre $V$ iSETRA, 1993), au sujet du frottement négatif.

\section{9}

\section{Influence de la cohésion du remblai}

La cohésion du matériau de l'épaisseur active h. intervient dans l'efficacité du renforcement. Le cas extrême d'une épaisse couche de grave ciment, mise directement en ceure sur les tètes d'inclusions, puis chargée, le prouve manifestement. Une difficulté subsiste cependant si l'on souhaite tenir compte de cette cohésion; elle doit être pérenne et homogène. Ainsi, une grave bien graduée, possédant un pourcentage de fines appréciable et compactée dans les meilleu. res conditions de teneur en eau, à l'optimum Proctor normal ou modifié, acquiert une cohésion quỉ peut s'avérer permanente : la situation de ce matériau en sous-couche du remblà est en effet favorable à une protection et au maintien de cette caractéristique. En outre, les tassements faibles que subira le remblai limitent beaucoup les risques de fissuration. En tout état de cause, la a valeur caractéristique y de la cohésion qui sera retenue pour justifier un ouvrage détinituf, devrá être prudente. Pour des chantiers expérimentaux ins-

rableau V Valeurs des contraintes $\mathrm{q}_{5}=q\left(\mathrm{~h}_{\mathrm{B}}\right)$, en $\mathrm{kPa}$, en fonction de la cohésion. Stresses $\mathrm{q}\left(\mathrm{h}_{\mathrm{a}}\right)$ with cohesion.

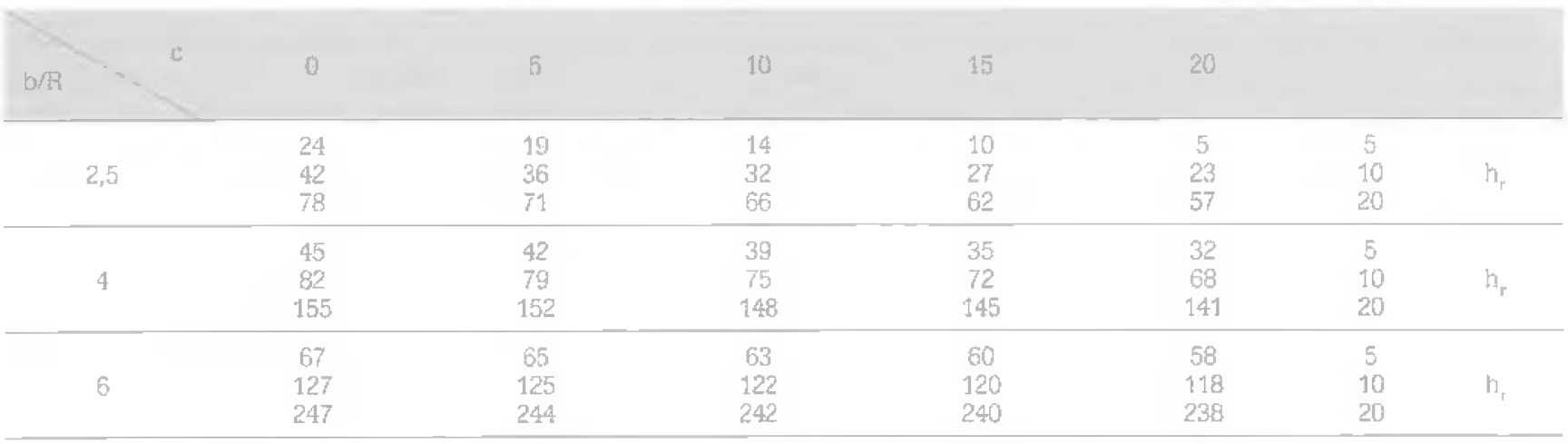


trumentés, par contre, la cohésion à retenir pour les interprétations sera la cohésion moyenne, en espérant une dispersion la plus faible possible.

L'introduction de la cohésion c mène pour la contrainte résicuelle q, à la valeur suivante

$q(s)=q\left(h_{n}\right)=\left(\frac{\gamma_{r}}{m_{r}}-\frac{c}{K \tan \varphi_{r}}\right)\left(1-e^{m h_{r}}\right)+\left(h_{r}-h_{n}\right) \gamma_{r} e^{-m h r}(8)$

Elle est inférieure à celle obtenue en (4) pour le sol purement frottant

On donne, dans le tableau $\mathrm{V}$, les résultats pour les trois hauteurs de remblai de 5,10 et $20 \mathrm{~m}$ avec des valeurs respectives de c : $5,10,15$ et $20 \mathrm{kPa}$.

On peut constater que la cohésion a d'autant moins d'influence que :

- l'espacement b/R augmente :

- l'épaisseur h augmente; d'ailleurs pour h croissant indéfiniment, le rappori SRR a, quelle que soit la colésion c, la même valeur que pour le sol simplement frottant.

Enfin, il est aisé de vêrifier que le upoids b de c est d'autant plus faible que $\mathrm{K}$ tan $\varphi$, est élevé. La cohésion n'a donc d'intérêt que pour des remblais de hauteurs modérées, tels les remblais routlers courants de 5 à $6 \mathrm{~m}$. Il est donc évident que tout procédé, permettant de conférer au matériau une cohésion permanente ả la partie active, va dans le sens, pour une efficacité fixée, d'un maillage un peu plus lâche pour les éléments rigides.

\section{Conclusion}

L'introduction, dans un remblai sur sol compressible renforcé par inclusions rigides, d’un plan d'égal tassement situé à une distance des têtes appelée hauteur active, conduit à modiffer l'expression donnant la valeur de la contrainte résiduelle en surface du sol initial. Cette valeur est plus forte que celle constituant la base de la méthode développée en 1988. La diminution de l'effort en tête d'inclusion est compensée par l'augmentation du frottement négatif dans la sol mou, et l'efficacité globale du groupe est peu affectée, pour les malliages relativement serrés. Enfin, on peut estimer que prévoir dans le sol porteur une pointe d'inclusion fixe ou, au contraire, susceptible de tasser, ne modifie guère le dimensionnement et le comportement de l'ouvrage. La qualité du remblai, appréciée par sor module, est déteminante dans l"efficacitẻ d"un groupe d'inclusions, et la cohésion vraie du remblai s'avère en outre bénéfique.

\section{$\overline{\text { Bibliographie }}$}

Combarieu O. - Amélioration des's sols par inclusions rigides verticales. Application a l'édification des rentblais sur sols médiocres. Revue française de géotech nique, $\Pi^{\circ} 44,1988$, p. $57-79$.

Combariew O. - Fondations superficielles sur sol amélioré par inclusions rigides, Revue française de géotechnique, $n^{\circ} 53$. 1990, p. $33-44$

Jenck 0. - Le renforcement des sols compressibles par inchusions rigides vertica-
Les. Modélisation physique et numérique Thèse UPGC de I"JNA de Lyon, 2005.

LCPC-COPREC - Caractéristiques des mátériax de renblai supports de fondgtions. Recommandations. 1980

Menard I. - Calcul de la capacitê portante des fondations sar la base des essais pressiométriques. Sols-Solls, ${ }^{\circ}$ 5, 1963, p. 9-28.
Menard 1 - Ancrages ànométre yaria bie. Notice D/93/69 du Centre d'études gêotechniques L Ménard, 1969.

SETPA - Fascicule 62 titre V. Rèlec techniques de conception et de calcul des fondations des ourrages de génie civil. CCTG, 1993

SETRA-ICPC - Réalisation des remblais at des cotidhes fe forme. Guide technigue. 1992 